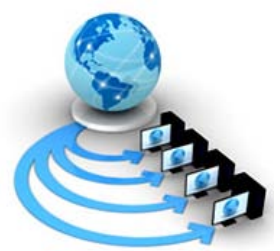

Volume 9, No. 2, March-April 2018

International Journal of Advanced Research in Computer Science

RESEARCH PAPER

\title{
PIL-LEACH MODEL FOR INCREASING ENERGY EFFICIENCY IN WIRELESS SENSOR NETWORK
}

\author{
Ram Charan Ponnada \\ Lovely Professional University \\ India
}

\begin{abstract}
Wireless Sensor Networks (WSN) play a vital role in the modern arena. WSN is deployed in the various vital areas such as military operations, health care, surveillance, home application and environmental applications. WSN mainly works on battery power, whose capacity is limited. Energy efficiency is one of the main issues in wireless sensor networks. Various routing techniques are developed to achieve maximum reliability, network lifetime and scalability. Leach is one of the clustering convention used in wireless sensor networks. Even, after its existence of more than 17 years, Leach protocol grabs attention and interest of research community. Our research area is confined to power management. This paper proposes a technique called as Improved Lifetime - Leach with Particle Swarm Optimization (PIL-LEACH) to smoothen the associated information transmission inside the group and, to control the problems such as correlated data, cluster head selection and large distance transmission of the packet in Leach. Particle Swarm Optimization (PSO) algorithm selects the cluster head based on various parameters of sensor nodes such as residual energy, distance from base sink etc. This approach admits that we know the area of the sensor hubs. It uses the two-tier architecture of clusters heads which are far away from the sink in order to mitigate the energy losses.
\end{abstract}

Keywords: Pil-Leach; PSO; Particle Swarm Optimization; Leach; Energy efficiency; Wireless Sensor Networks

\section{INTRODUCTION}

Wireless Sensor Network is a collection of the base station and multiple sensor nodes, which are deployed to sense and collect different types of data. Sensor nodes consist various units like sensors, micro-controller, memory, microprocessor, antenna, battery, etc[1]. Sensor unit sense and collects data like humidity, pulse, temperature, pressure, etc. These nodes usually tend to face some serious problems like resource constraints and energy constraints due to deployment conditions and their size. The WSN is usually deployed, where the wired deployment is not possible due to various reasons like harsh hostile environmental conditions, emergencies, secrecy, etc. The major deployment areas of WSN are troops tracking, healthcare, [2] border monitoring, forest fire detection, earthquake detection, rescue operations, etc. Nodes anchored in such locations, over a period of time, may not be accessible physically after their post-deployment to repair its internal components which are a big hurdle to maintain wireless sensor network. Hence there is a need to have an efficient energy mechanism to transmit the data for a large period of time. A lot of many good effective techniques were developed for aggregating the sensed data and forward the sink [3]. The prominent and efficient one among such routing mechanisms is Low Energy Adaptive Clustering Hierarchy.

LEACH stands for Low-Energy Adaptive Clustering Hierarchy [10].LEACH is a dynamic clustering routing protocol for WSN. All nodes are homogenous sensor nodes. All nodes participate in cluster head selection [7], a single node will be selected as a cluster head by a random probability function [8]. Nodes transmit data to their cluster head. Cluster head aggregates the data and sends it to sink. It increases the energy efficiency by randomly selecting $\mathrm{CH}$.
Leach has two phases:

- $\quad$ Set-Up Phase

1. Nodes forms clusters.

2. All nodes participate in the cluster head selection.

3. A single node is selected as Cluster-Head.

- $\quad$ Steady Phase

1. Cluster-Head is maintained.

2. Data is transmitted between nodes.

Advantages of leach:

1. Cluster head schedules [9] Time Division Multiple Access (TDMA) slots to every node, as a result, there will be no collision among nodes and nodes will go into sleep mode till it gets TDMA schedule, as a result, the battery can be saved.

2. LEACH protocol provides an equivalent chance to all sensor nodes to become the cluster head at least once and for a member node several times during its lifetime.

Problems in leach:

1. In LEACH, a cluster head is selected randomly. There is a probability to select a node that, have less energy as a cluster head. Due to this, a lifetime of network drastically affected. 
2. Leach will not guarantee the number .of cluster heads $(\mathrm{CH})$ and position of $\mathrm{CH}$. As a result, load balancing will not be guaranteed.

Due to its single-level communication, between cluster head $(\mathrm{CH})$ and the base station, a $\mathrm{CH}$ which is far away from base station have to consume more energy for sending data which leads to uneven energy consumption. This reduces the lifetime of WSN [4], [5], [6].

In this paper, we have used PSO algorithm to get the global best of entire nodes and will use this calculate fitness function, to select cluster head selection.

\section{LITERATURE REVIEW}

Wendi B. Hein Zelman et al. [10], developed low-energy adaptive clustering hierarchy (LEACH), a protocol for wireless sensor networks, which is a combination of the energy-efficient cluster-based routing and media access protocols, in order to attain good performance in terms of network lifetime, latency. This technique provides mechanisms to form cluster and rotation of cluster heads to minimize the energy dissipations as well as reduces, the load on cluster head. The importance of considering, the function of the application, the necessity for ease of distribution, and the severe energy limitations of the nodes, leads to design LEACH. In which the computations are performed locally in view of reducing the volume of transmitted data. The network configuration and media access control and routing protocols are done locally which enables to maintain a lowenergy network.

Sunil Kumar Singh et al. [11] studied the different variants of LEACH Protocols proposed up, until this point and talks about the improvement and working of them. He classifies all the successor of leach protocols into two type's namely multi-hop and single hop based on the communication from cluster head to sink. He analyses more than sixty variants of leach on various parameters such as complexity, energy efficiency, overhead and scalability along with its pros and cons of each successor of leach. Most of the recently developed protocols are focused on energy efficiency over the other performance factors. It was observed that a very less number of protocols considered the energy consumption during cluster head selection and cluster formation. And researchers considered various other parameters such as the optimal number of $\mathrm{CHs}$, the location of the node, mobility, node density, energy harvesting nodes at the cluster head selection process. Protocols proposed for security in WSN are mainly concentrated to develop security and energy efficiency side by side. In modern years the deterministic approach of clustering has gained the researcher's eye, but the development of deterministic approach clustering procedures increase the complexity and the energy consumption when compared to probabilistic approach.it was because of usage of different approaches like compound based, fuzzy-logic based, weight-based in deterministic clustering.
Firoj Ahamad et al. [12], proposed a method to extend the WSN lifetime by means of fuzzy logic based selection of $\mathrm{CH}$ which provides an absolutely non-conventional probabilistic methodology. This methodology uses the residual energy of the node and the distance from the sink, as two fuzzy variables. Multi-hop communication was used for data transmission. The cluster head is authorized to communicate with both sink and other cluster heads. The results are convincing that the proposed methodology extended the network lifetime by selecting proper nodes as a cluster head. He also stated that the future scope will be selecting cluster head in sub-areas on demand dynamically, rather than selecting the cluster head after some time which is an energy consuming process. He also stated that, in order to meet the dynamic on-demand cluster head selection, artificial intelligence can be used.

Santa Pal Singh et al. [13], proposed a methodology based on Particle Swarm Optimization (PSO).The cluster head is selected by PSO on basis of its maximum power of nodes. The algorithm is a combination of proficient particle encoding method and fitness function. The algorithm significantly increases the network lifetime, by balances the energy consumption of cluster heads. The proposed methodology performs well in terms of network lifetime, packet delivery ratio, throughput etc. when compared with other algorithms.

Alka Singh et al. [14], proposed V-LEACH protocol in which, he introduced vice cluster head. If due to some condition cluster head fails due to lack of energy, vice cluster head will now take charge of the cluster head. The cluster head was selected by Particle Swarm Optimization technique based on residual energy, distance from the sink, velocity etc. In comparison with existing leach, the $\mathrm{V}$ LEACH performs very well in terms of energy dissipations and networks lifetime.

Rajat Kandpal et al. [15], proposed IL-LEACH (Improved Lifetime- Low Energy Adaptive Clustering Hierarchy). This is an enhancement to existing LEACH protocol. Sensor nodes that are placed in neighborhood collect correlated data then sent to the $\mathrm{CH}$. The main aim of IL-LEACH is to mitigate the redundant data transmissions in the cluster with the formation of the virtual correlated cluster (VCC) and letting only a single node to transmit data in that TDMA slot. It mitigates and suppresses the co-related data transmissions, which are redundant and drains sensor nodes energy. As a result, it increases the network lifetime and reduces the congestion in the network. 


\section{Methodology}

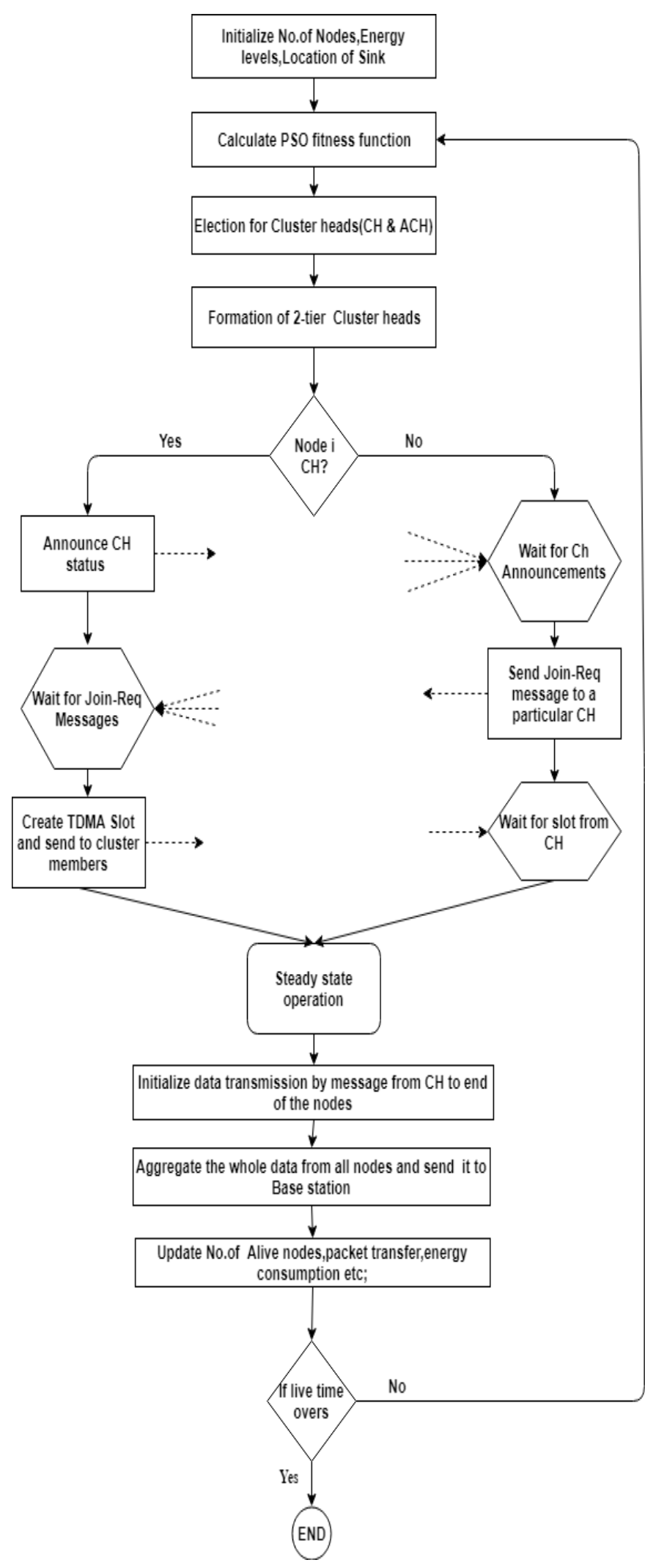

Figure 1 PIL-LEACH algorithm
We have proposed a model called PIL-LEACH (Increased Life efficiency of Low-Energy Adaptive Clustering Hierarchy by particle swarm optimization). We assume that all sensor nodes are homogenous nature in terms of capability, power etc. Here we have used cluster head and advanced cluster head. The cluster head is selected by election probability, which uses the fitness value calculated by particle swarm optimization. The PIL-LEACH has two phases, they are set-up phase and steady phase.

\section{- Set-Up Phase:}

1. Initialization will be carried by defining the deployment area, location of sink, number of nodes, initial energy of nodes etc.

2. Fitness value for a node will be calculated by particle swarm optimization.

3. Fitness values are calculated on various parameters such as energy levels of nodes, the velocity of nodes and location of nodes.

4. The nodes which do not meet the average residual energy of all nodes will be kept out from the election.

5. Nodes are selected as cluster heads (cluster head \& advanced cluster heads) by-election probability, which takes the fitness value as input.

6. Advanced $\mathrm{CH}$ has high energy level when compared to $\mathrm{CH}$.

7. The selected cluster heads ( $\mathrm{CH} \& \mathrm{ACH})$ forms a two-tier architecture network.

8. The $\mathrm{CH}$ announce its status to other sensor nodes a within its vicinity range and waits for join request from other sensor nodes.

9. The nodes wait for $\mathrm{CH}$ announcements and send a join request to $\mathrm{CH}$ that is in its vicinity range.

10. These nodes (Normal and $\mathrm{CH}$ ) forms a cluster and $\mathrm{CH}$ schedules TDMA slot for each node in the cluster.

- Steady Phase:

1. In steady phase, the cluster formation is maintained.

2. Nodes start transmitting data to cluster head in their scheduled TDMA slot.

3. The cluster head aggregates the data from all the sensor nodes and transmits it to the base station.

Both the phases are carried out till the network lifetime completes. 


\section{SIMULATION RESULTS}

We took an area of $100 * 100$ (length*breadth) for network deployment. Initially, the nodes are scattered randomly.

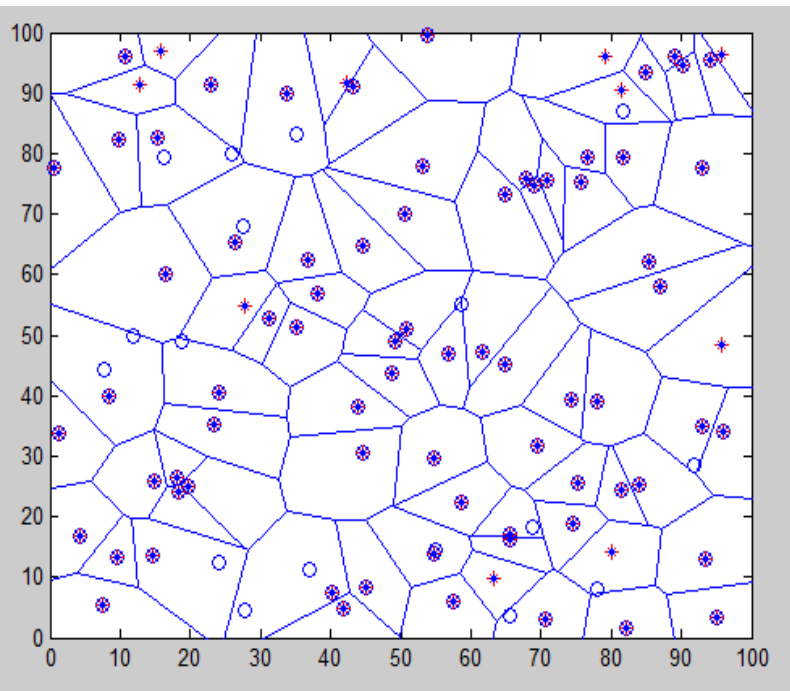

Figure 2 Sensor Network when nodes are alive

Later clusters were formed along with cluster heads. Cluster heads and cluster are changed regularly on new fitness values.

When the dead nodes fall to ninety percent of overall nodes, the network is called to be dead.

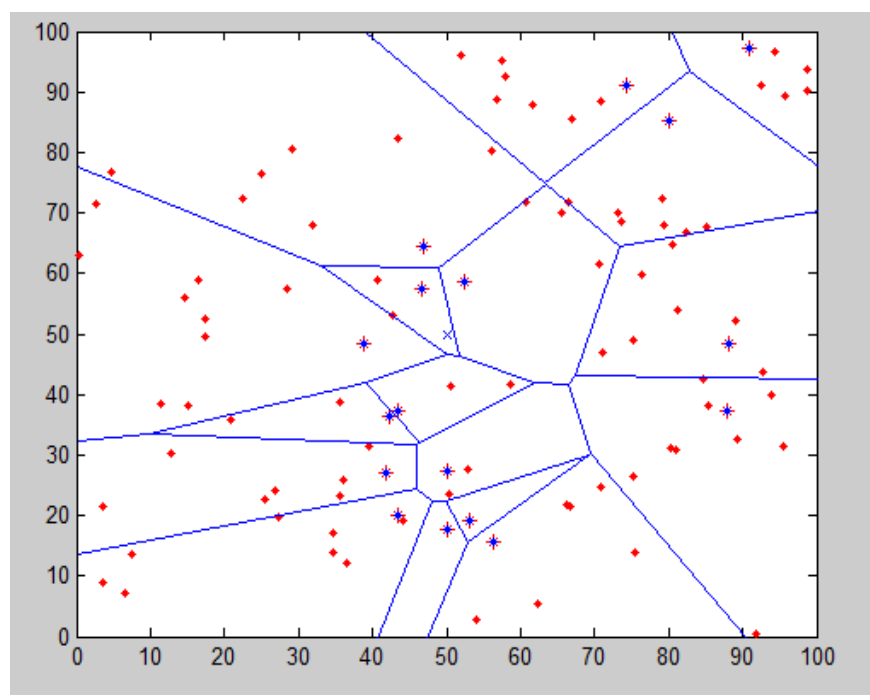

Figure 3 Sensor Network when nodes are dead

The dead nodes are represented by red dots. There are some nodes which are represented by star symbol, these advanced cluster head nodes.

The below figure shows the efficiency of MLEACH. The xaxis represents the number of rounds, whereas the y-axis represents the number of dead sensor nodes. Here the first nodes dead's at 1000 'Th round and the last node dead's at 3000 'Th round approximately. The sensor network is stable from 1000 to 1500 'Th round. After that, the performance of network gradually decreases. At 3000'Th round, the network dead's complete.

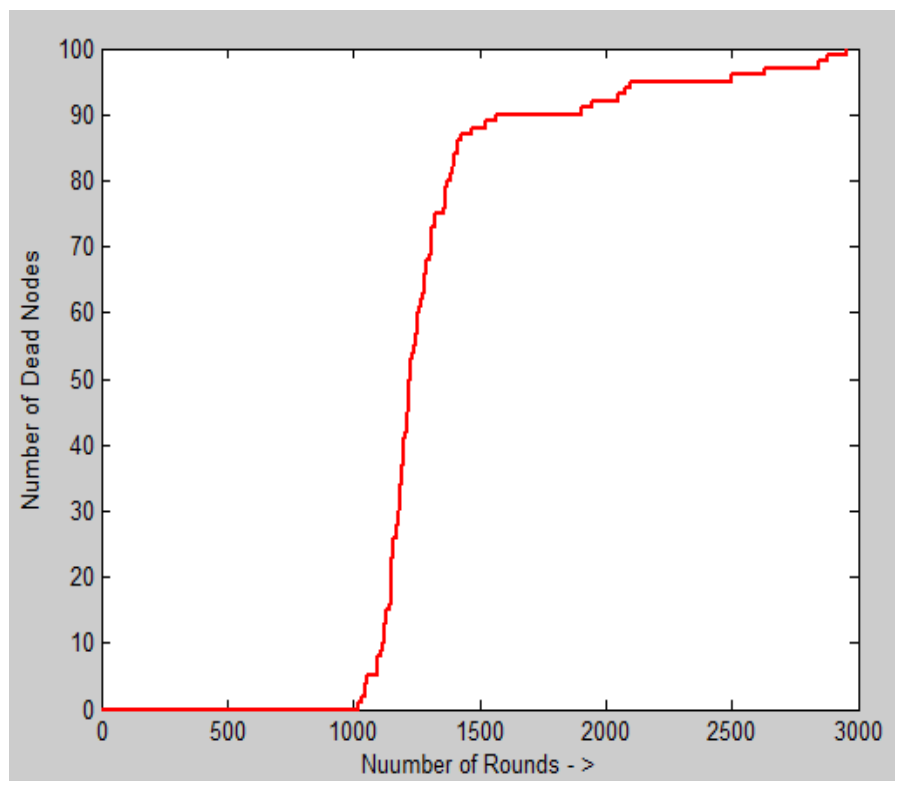

Figure 4 Mleach performance

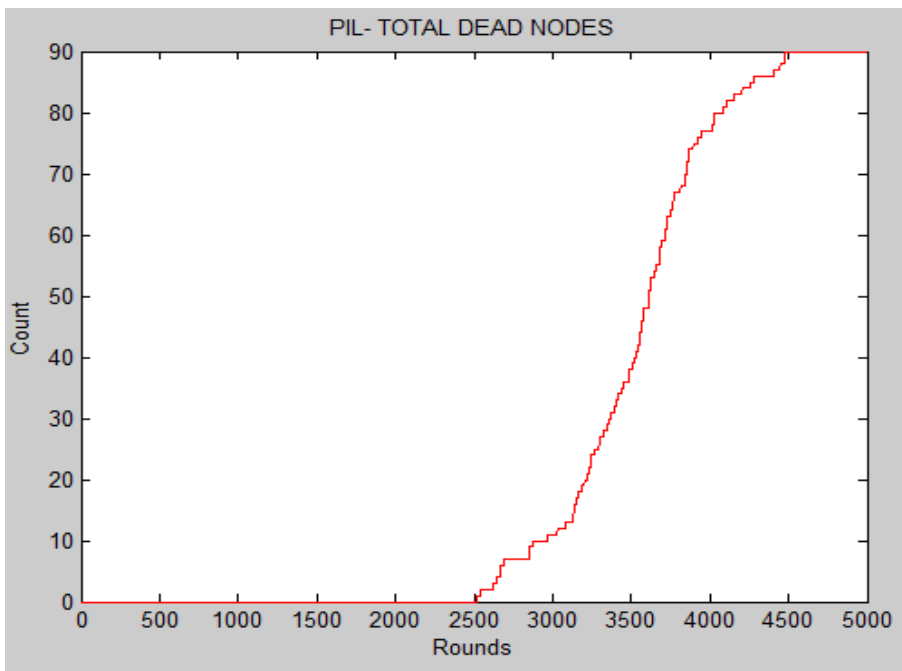

Figure 5 PIL-LEACH performance

The above figure shows the efficiency of PIL-LEACH. The $\mathrm{x}$-axis represents the number of rounds, whereas the y-axis represents the number of dead sensor nodes. Here the first nodes dead's at 2500 'Th round and the last node dead's at 4500 'Th round approximately. PIL-LEACH performs well when compared to MLEACH

\section{CONCLUSION}

This paper is presented on Improved Lifetime - LEACH with Particle Swarm Optimization (PIL-LEACH) to smother the associated information transmission inside the group and, to control the problems such as correlated data, cluster head selection and large distance transmission of the packet in Leach. PSO algorithm selects the cluster head based on various parameters of sensor nodes such as residual energy, the velocity of node and location of the node. It uses the 
two-tier architecture of clusters heads network, in order to mitigate the energy losses involved in far distance data transfer. It performs well when compared to existing protocols in terms of network lifetime. The future scope will be selecting cluster head dynamically whenever it is needed, rather than selecting the cluster head after some time which is an energy consuming process and in order to meet the dynamic on-demand cluster head selection, artificial intelligence can be used.

\section{REFERENCES}

[1] Holms, Ann, and Ethan Culler-Mayeno. "A Technical Report: Wireless Sensor Networks and How They Work." University of California Santa Barbara (2006).

[2] Puccinelli, Daniele, and Martin Haenggi. "Wireless sensor networks: applications and challenges of ubiquitous sensing." IEEE Circuits and systems magazine 5.3 (2005): 19-31.

[3] Ramesh, K., and Dr. K. Somasundaram. "A comparative study of cluster head selection algorithms in wireless sensor networks." arXiv preprint arXiv:1205.1673 (2012).

[4] Kumar, Ajay, Vikrant Sharma, and D. Prasad. "Distributed deployment scheme for homogeneous distribution of randomly deployed mobile sensor nodes in wireless sensor network." Editorial Preface 4.4 (2013): 139-146.

[5] Dutt, Suniti. "Cluster based Hierarchical Routing in wireless sensor networks." International journal of Engineering and innovative technology (IJEIT) vol 2 (2012).

[6] Paul, Sounak, and Tapan Kumar Dey. "Energy efficient routing in cluster based wireless sensor networks." Advanced Informatics: Concepts, Theory and Applications (ICAICTA), 2015 2nd International Conference on. IEEE, 2015.

[7] Mazumdar, Nabajyoti, and Hari Om. "Distributed energyefficient clustering algorithm for mobile-sink based wireless sensor networks." Intelligent Systems and Control
(ISCO), 2016 10th International Conference on. IEEE, 2016.

[8] Amsalu, Seifemichael B., et al. "Energy efficient Grid Clustering Hierarchy (GCH) routing protocol for wireless sensor networks." Ubiquitous Computing, Electronics \& Mobile Communication Conference (UEMCON), IEEE Annual. IEEE, 2016.

[9] Kaur, Navdeep, Dalveer Kaur Grewal, and Shashi Kant Shankar. "Typical and atypical hierarchical routing protocols for WSNs: A review." Computing, Communication and Automation (ICCCA), 2016 International Conference on. IEEE, 2016.

[10] Heinzelman, Wendi B., Anantha P. Chandrakasan, and Hari Balakrishnan. "An application-specific protocol architecture for wireless microsensor networks." IEEE Transactions on wireless communications 1.4 (2002): 660670.

[11] Singh, Sunil Kumar, Prabhat Kumar, and Jyoti Prakash Singh. "A survey on successors of LEACH protocol." IEEE Access 5 (2017): 4298-4328.

[12] Ahamad, Firoj, and Rakesh Kumar. "Energy efficient region based clustering algorithm for WSN using fuzzy logic." Recent Trends in Electronics, Information \& Communication Technology (RTEICT), IEEE International Conference on. IEEE, 2016.

[13] Singh, Santar Pal, and Subhash Chander Sharma. "A Particle Swarm Optimization Approach for Energy Efficient Clustering in Wireless Sensor Networks." International Journal of Intelligent Systems and Applications 9.6 (2017): 66.

[14] Singh, Alka, Shubhangi Rathkanthiwar, and Sandeep Kakde. "Energy efficient routing of WSN using particle swarm optimization and V-LEACH protocol." Communication and Signal Processing (ICCSP), 2016 International Conference on. IEEE, 2016.

[15] Kandpal, Rajat, and Rajesh Singh. "Improving lifetime of wireless sensor networks by mitigating correlated data using LEACH protocol." Information Processing (IICIP), 2016 1st India International Conference on. IEEE, 2016. 